\title{
Mitogen- and stress-activated Kinase 1 mediates Epstein-Barr virus latent membrane protein 1-promoted cell transformation in nasopharyngeal carcinoma through its induction of Fra-1 and c-Jun genes
}

Binbin $\mathrm{Li}^{1,2+}$, Zheng Wan ${ }^{2 \dagger}$, Guoliang Huang ${ }^{2}$, Zunnan Huang ${ }^{2}$, Xiangning Zhang ${ }^{1}$, Dan Liao ${ }^{1}$, Shengqun Luo ${ }^{1}$ and Zhiwei $\mathrm{He}^{1,2^{*}}$

\begin{abstract}
Background: Mitogen- and Stress-Activated Kinase 1 (MSK1) is a nuclear kinase that serves as active link between extracellular signals and the primary response of gene expression. However, the involvement of MSK1 in malignant transformation and cancer development is not well understood. In this study, we aimed to explore the role of MSK1 in Epstein-Barr virus (EBV) latent membrane protein 1 (LMP1)-promoted carcinogenesis of nasopharyngeal carcinoma (NPC).

Methods: The level of MSK1 phosphorylation at Thr581 was detected by the immunohistochemical analysis in NPC tissues and normal nasopharynx tissues, and its correlation with LMP1 was analyzed in NPC tissues and cell lines. Using MSK1 inhibitor H89 or small interfering RNA (siRNA)-MSK1, the effects of MSK1 on LMP1-promoted CNE1 cell proliferation and transformation were evaluated by CCK-8 assay, flow cytometry and focus-forming assay respectively. Furthermore, the regulatory role of MSK1-mediated histone H3 phosphorylation at Ser10 on the promoter activity and expression of Fra-1 or c-Jun was determined by reporter gene assay and western blotting analysis.

Results: Immunohistochemical analysis revealed that the level of MSK1 phosphorylation at Thr581 was significantly higher in the poorly differentiated NPC tissues than that in normal nasopharynx tissues $(P<0.001)$. Moreover, high level of phosphorylated MSK1 was positively correlated with the expression of LMP1 in NPC tissues $(r=0.393, P=0.002)$ and cell lines. MSK1 inhibitor H89 or knockdown of MSK1 by siRNA dramatically suppressed LMP1-promoted CNE1 cell proliferation, which was associated with the induction of cell cycle arrest at $G_{0} / G_{1}$ phase. In addition, the anchorage-independent growth promoted by LMP1 was blocked in MSK1 knockdown cells. When the activity or expression of MSK1 was inhibited, LMP1-induced promoter activities of Fra-1 and c-Jun as well as their protein levels were greatly reduced. It was found that only H3 WT, but not mutant H3 S10A, dramatically increased LMP1 induction of Fra-1 and c-Jun genes compared with mock cells.

(Continued on next page)
\end{abstract}

\footnotetext{
* Correspondence: zhiweihe688@yahoo.com

${ }^{\dagger}$ Equal contributors

'Department of Pathophysiology, Guangdong Medical College, Dongguan, Guangdong 523808, China

${ }^{2}$ Key Laboratory for Medical Diagnostics of Guangdong Province,

Sino-American Cancer Research Institute, Guangdong Medical College,

Dongguan, Guangdong 523808, China
} 
(Continued from previous page)

Conclusion: Increased MSK1 activity is critically important for LMP1-promoted cell proliferation and transformation in NPC, which may be correlated with its induction of Fra-1 and c-Jun through phosphorylation of histone $\mathrm{H3}$ at Ser10.

Keywords: MSK1, NPC, EBV-LMP1, Transformation, Fra-1, c-Jun

\section{Background}

Nasopharyngeal carcinoma (NPC) is a particularly common tumor in areas of southern China and South-East Asia, reaching a peak incidence of 20-30 cases per 100,1000 per year [1]. Its occurrence involves the interaction of host genetic alterations with environmental factors, especially infection by Epstein-Barr virus (EBV) $[2,3]$. EBV-encode latent membrane protein 1 (LMP1) is essential for the maintenance of latent infection and EBVmediated malignant transformation [3, 4]. The C-terminal tail of LMP1 provides docking sites for the recruitment and activation of signaling adapter proteins, which triggers various downstream oncogenic signaling pathways, such as NF-kB, MAPK, PI3K and JAK/STAT pathways [5]. However, it is largely unknown what key effector proteins are induced and essentially promote cell transformation in the LMP1-triggered signaling events.

Mitogen- and stress-activated kinase 1 (MSK1) is a nuclear serine/threonine protein kinase that is mainly activated by both ERKs and p38 MAPKs. Because of its dual activation mode, MSK1 is able to integrate various physiological and pathological stimuli including mitogenic signals, cellular stress and pro-inflammatory cytokines [6]. MSK1 has been recognized as a versatile kinase regulating gene transcription at multiple levels. MSK1 directly phosphorylates various transcription factors, including cAMP-responsive element binding protein (CREB), activating transcription factor 1 (ATF1), signal transducers and activators of transcription 3 (Stat3), the p65 subunit of $\mathrm{NF}_{-\mathrm{K}} \mathrm{B}$, thereby alters their ability of binding to target DNA or recruitment of required coactivator [7-9]. MSK1 also mediates the phosphorylation of chromatin protein histone $\mathrm{H} 3$ and high mobility group 14 (HMG14), which induces chromatin relaxation and contributes to gene activation, termed as "nucleosomal response" [10]. MSK1-mediated histone H3 phosphorylation at Ser10 is critical for epidermal growth factor (EGF), 12-O-tetradecanoylphorbol-13-acetate (TPA) and oncogene (e.g. H-ras)-induced cell transformation, which is associated with the induction of immediate-early (IE) genes such as c-Fos, $c$-Jun and $c-M y c$ [11-13]. Overactive Ras-MAPK pathway and elevated MSK1 activity were observed in various cancerous tissues and cell lines $[14,15]$. MSK1 is responsible for histone H3 phosphorylation of estrogen-responsive Trefoil Factor-1 (TFF-1) promoter in breast cancer MCF-7 cells [16]. MSK1 also regulates transcriptional activation of $\mathrm{NF}_{-} \mathrm{B}$-dependent pro-inflammatory genes in response to cigarette smoke [17]. These studies suggested that MSK1 might play an important role in carcinogenesis through aberrant histone modifications and transcriptional regulation. Our previous study demonstrated that phosphorylation of histone $\mathrm{H} 3$ at Ser10 mediated by MSK1 might be a crucial epigenetic change in LMP1-promoted carcinogenesis of NPC [18]. However, it is not completely clear whether the activation of MSK1 directly affects LMP1-promoted cell proliferation and transformation in NPC.

In the present study, we found that MSK1 was abnormally activated in both primary NPC tissues and NPC cell lines, and closely related to the expression of LMP1. Moreover, MSK1 activity was required for LMP1-promoted cell proliferation and transformation of NPC, which was associated with the induction of Fra-1 and c-Jun by phosphorylation of histone $\mathrm{H} 3$ at Ser10. These findings provide a better understanding to the importance of MSK1-mediated nucleosomal response in the LMP1-induced malignant transformation and carcinogenesis.

\section{Methods}

\section{Patients, tissue specimens and cell lines}

Nasopharyngeal carcinoma tissue microarray (catalog no. NPC961) was from US Biomax (Rockville, MD), including 33 cases of poorly differentiated NPC tissues, 26 cases of adjacent normal tissues, and 10 cases of normal nasopharyngeal tissues. In addition, 20 cases of poorly differentiated NPC tissues were obtained from the First Affiliated Hospital of Guangdong Medical College, Zhanjiang, China. The patients received no other therapies, such as radiation or chemotherapy, prior to operation. All samples were confirmed by pathological examination and staging was performed according to the 1997 NPC staging system of the UICC. In the 53 NPC cases, there were 40 male and 13 female with age ranging from 26 to 62 years (median, 43.9 years). Informed consent was obtained from all patients, and this study was approved by the Institutional Ethics Committee of Guangdong Medical College.

CNE1 cells, an EBV-negative and well-differentiated human NPC cell line, were cultured in RPMI 1640 medium supplemented with $10 \%$ fetal bovine serum (GIBCO, Carlsbad, CA, USA). CNE1G (CNE1 stably transfected with PAT-GFP) and CNE1GL (CNE1 stably transfected with PAT-GFP-LMP1) cells were provided by Dr. Xiaoyi Chen, Guangdong Medical College [19], and 
were maintained in completed RPMI 1640 medium described above, containing $0.5 \mu \mathrm{g} / \mathrm{ml}$ puromycin (SigmaAldrich, St. Louis, MO, USA).

\section{Plasmids, transfection and establishing stable cell lines} To construct the siRNA-mock (si-mock) or siRNA-MSK1 (si-MSK1), the mU6pro vector (a gift from Dr. Zigang Dong, Hormel Institute, University of Minnesota, Austin, Minnesota, USA) was digested with XbaI and BbsI. The annealed synthetic primers (si-mock: 5'-TTTGACTAC CGTTGTTATAGGTGTTCAAGAGACACCTATAACAA CGGTAGTTTTTT-3' and antisense 5' - CTAGAAAAAA ACTACCGTTGTTATAGGTGTCTCTTGAACACCT AT AACAACGGTAGT; si-MSK1: sense 5'-TTTGAGACCT AATTCAGCGTCTTTTCAAG AGAAAGACGCTGAAT TAGGTCTTTTTT-3' and antisense 5' -CTAGAAAAAA GACCT AATTCAGCGTCTTTCTCTTGAAAAGACGC TGAATTAGGTCT-3') were then introduced following the recommending protocols. The recombinant plasmids were confirmed by agarose gel electrophoresis and DNA sequencing. The plasmids were transfected into CNE1 cells using JetPEI (Polyplus, llkirch) according to the manufacturer's protocol. Stable CNE1 cells expressing si-mock or si-MSK1 were established with pcDNA6.0/myc-HisB as selection marker. Transfected cells were selected in medium containing $2 \mu \mathrm{g} / \mathrm{ml}$ blasticidin (Sigma-Aldrich, St. Louis, MO), and the expression level of MSK1 was confirmed by Western blotting analysis.

The pcDNA3.0 and pcDNA3.0-LMP1 vectors were kindly provide by Dr Ellen Cahir- McFarland, Brigham and Women's Hospital, Boston, Massachusetts, USA. AP-1 reporter vector pRTU14 was kindly provided by Dr ArndKieser, Helmholtz ZentrumMünchen, Munich, Germany [20]. To construct the Fra-1 and c-Jun promoter luciferase reporter vectors, DNA fragments of 5 '-flanking region of the human Fra-1 gene (-379 to -238) [21] and c-Jun gene (-117 to -50) [22] were synthesized and inserted into a basal promoter luciferase reporter vector (pGL3) respectively. The pcDNA6.0/myc-His B-histone H3 wide-type (pcDNA6.0-H3 WT) and pcDNA6.0/mycHis B-histone H3 S10A mutant (pcDNA6.0-H3 S10A) were constructed as reported previously [18]. pcDNA6.0H3 WT or pcDNA6.0-H3 S10A stably transfected CNE1 cells were selected in medium containing $2 \mu \mathrm{g} / \mathrm{ml}$ blasticidin. Expression of vectors was confirmed with an antibody against the His epitope by Western blotting analysis.

\section{Immunohistochemical staining}

Fixed tissue samples were sectioned $(4 \mu \mathrm{m})$, deparaffinized, rehydrated, and subjected to heat-induced antigen retrieval in sodium citrate buffer (0.01 M, pH 6.0). Endogenous peroxidase activity and non-specific antigen were blocked with $3 \%$ hydrogen peroxide and normal goat serum. The sections were incubated with the primary antibodies against phosphorylated MSK1 (Thr581) or LMP1 overnight at $4{ }^{\circ} \mathrm{C}$. HRP-conjugated secondary antibodies (ChemMate Envision Detection Kit, DAKO) were applied onto the sections and incubated for $30 \mathrm{~min}$ at room temperature. $10 \%$ normal goat serum was used to replace primary antibodies as a negative control.

The slides were reviewed and scored independently by two pathologists. Phosphorylated MSK1 at Thr581 was expressed in the cell nucleus and the staining was scored according to its intensity ( 0 , no staining; 1 , weakly staining; 2, moderately staining; 3 , strongly staining) and the percentage of positive cells $(0,<5 \%$ positive cells; $1,5 \%-25 \%$ positive cells; $2,26-50 \%$ positive cells; $3,>50 \%$ positive cells). The final expression score was calculated from "intensity score" multiplied by "percentage". For statistical analysis, cases with weighted scores of more than 3 were defined as high expression, otherwise they were defined as low expression. Staining of LMP1 appeared on the cell membrane or/and in the cytoplasm. The expression of LMP1 was scored as positive $(\geq 10 \%)$ and negative $(<10 \%)$ based on the percentage of stained cells [23]. In order to detect the expressions of LMP1 and phosphorylated MSK1 at Thr581 in CNE1G and CNE1GL cells, they were immunocytochemically stained using the same method as for the clinical specimens.

\section{Cell proliferation assay}

CNE1G and CNE1GL cells were seeded in 96-well plates $\left(2 \times 10^{3}\right.$ cells per well $)$ and incubated with different concentrations of H89 (Cell Signaling Technology, Beverly, MA). The si-mock or si-MSK1 stable CNE1 cells were transfected with pcDNA3.0 or pcDNA3.0-LMP1 plasmid and then seeded in 96-well plates with $100 \mu \mathrm{l}$ cell suspension in each well $\left(1 \times 10^{3}\right.$ cells per well). After culturing for various periods of time, $10 \mu \mathrm{l}$ of CCK- 8 solution (Dojindo Laboratories, Kumamoto, Japan) was added to each well, and cells were then incubated for 4 hour at $37{ }^{\circ} \mathrm{C}$. Absorbance was measured at $450 \mathrm{~nm}$ using Synergy2 Multi-Mode Microplate Reader (BioTek, Winooski, VT, USA). The assay was conducted in five replicate wells for each sample and three parallel experiments were performed.

\section{Cell cycle analysis}

CNE1 cells were seeded in 6-well plates and then transiently transfected with pcDNA3.0 or pcDNA3.0-LMP1 plasmid. H89 was added to the culture medium with different concentrations every $12 \mathrm{~h}$ after transfection. The si-mock or si-MSK1 stable CNE1 cells were transfected with pcDNA3.0 or pcDNA3.0-LMP1 plasmid and cultured for $48 \mathrm{~h}$. The cells were harvested with trypsin, fixed with $70 \%$ ice-cold ethanol, and stained with propidium iodide (PI). Then cell cycle distributions were analyzed by flow cytometry and the percentage of cells in $\mathrm{G}_{1} / \mathrm{G}_{0}, \mathrm{~S}$, or $\mathrm{G}_{2} / \mathrm{M}$ phase was calculated using ModFit 
LT software. Data represent the mean value derived from triplicate experiments.

\section{Colony forming assay}

CNE1G and CNE1GL cells were seeded in 6-well plates (400 cells per well) and incubated with different concentrations of H89. After culturing for 2 weeks, colonies were fixed with methanol and stained with $0.5 \%$ crystal violet. The number of colonies containing 50 cells or more was counted under an inverted microscope and the mean value from three replicate wells was calculated. Data are representative of at least three independent experiments.

\section{Anchorage-independent cell transformation assay}

LMP1-induced cell transformation was investigated in si-mock or si-MSK1 stable CNE1 cells. In brief, the cells were transfected with pcDNA3.0 or pcDNA3.0-LMP1 plasmid and then seeded in $1 \mathrm{ml}$ of $0.3 \%$ basal medium Eagle's agar containing 10 \% FBS (800 cells per well). After culturing for 3 weeks, the number of colonies containing 50 cells or more was counted under an inverted microscope and the mean value from three replicate wells was calculated. Data are representative of at least three independent experiments.

\section{Reporter gene assay}

Fra-1 or c-Jun promoter activity and AP-1 activity were determined by the luciferase reporter gene assay. Cells were transiently cotransfected with various firefly luciferase reporter gene and pRL-TK vector (Promega, China). The pRL-TK vector expressing Renilla luciferase was cotransfected for normalizing the transfection efficiency. At 36 hours after transfection, cells were lysed with passive lysis buffer (Promega) for $20 \mathrm{~min}$ with gently shaking. The firefly luciferase and Renilla luciferase activities were measured with cell lysates using the Dual-Luciferase assay system (Promega) in FB12 Luminometer (Berthold detection system). The results were expressed as firefly luciferase activity normalized against Renilla luciferase activity. Data were derived from the mean value of triplicate samples and recorded as relative luciferase activity (fold or \%). All experiments were done at least in triplicate.

\section{Protein extraction and western blot analysis}

Cells were washed twice with cold PBS, and total protein was extracted with RIPA lysis buffer (Beyotime Ins. Bio, China) with protease inhibitor PMSF (Sangon, China). The isolation of histone was performed as described previously [18]. Protein concentration was determined by the bicinchoninic acid (BCA) assay (Pierce, Rockford, IL, USA). Samples containing equal amount of protein were resolved by SDS-PAGE and transferred to PVDF membranes. The membranes were probed with primary antibodies against phosphorylated or total MSK1(1:500), c-Jun
(1:500), His-tag (1:500), histone H2A (1:1000) (all from Cell Signaling Technology, Beverly, MA), Fra-1(1:500, Santa Cruz Biotechnology, CA) and EBV LMP1 (CS1-4) (1:1000, DAKO, Glostrup), then incubated with infrareddye-conjugated secondary antibodies (1:10000, Rockland Immunochemicals, Gilbertsville, PA) for $1 \mathrm{~h}$ at room temperature. Protein bands were visualized by Odyssey Infrared Imaging System (LI-COR Biotechnology, Lincoln, NE, USA) and quantified using the Quantity One software (Bio-Rad, USA).

\section{Statistical analysis}

The SPSS version 16.0 software package and GraphPad Prism were used for the statistical analysis and data plotting. The Chi-square test was applied to examine the difference of phosphorylated MSK1 between NPC tissues and normal nasopharynx tissues as well as the relationship between phosphorylated MSK1 and LMP1 expression. Quantitative values were expressed as means \pm SD. Student $t$-test was used to compare the mean value of each group. $P<0.05$ was considered statistically significant.

\section{Results}

The level of MSK1 phosphorylation at Thr581 and its correlation with LMP1 in NPC tissues

In order to assess the activation of MSK1 in the tumorigenesis of NPC, we analyzed the level of MSK1 phosphorylation at Thr581 in 53 archived paraffin-embedded NPC specimens and 36 adjacent/normal nasopharynx specimens using immunohistochemical staining. Phosphorylated MSK1 was expressed in cell nucleus (Fig. 1a, b). As shown in Table 1, the level of MSK1 phosphorylation at Thr581 was significantly higher in the poorly differentiated NPC tissues than that in normal nasopharynx tissues $\left(X^{2}=15.137, P<0.001\right)$. This revealed that the elevated phosphorylation of MSK1 at Thr581 might be involved in the malignant transformation of NPC cells.

We further determined the relationship between phosphorylated MSK1 at Thr581 and LMP1 expression in 53 cases of NPC specimens. The LMP1 expression was located on cell membrane and cytoplasm (Fig. 1c, d). In NPC, 30 out of 53 (56.6 \%) cases showed LMP1 expression. As shown in Table 2, there was a positive correlation between LMP1 expression and increased phosphorylation of MSK1 at Thr581 in NPC tissues $(r=0.393, P=0.002)$.

\section{LMP1 induces MSK1 activation via ERK-MAPK pathway in CNE1 cells}

To investigate whether LMP1 directly induced activation of MSK1 in NPC cells, we examined the relative level of phosphorylated MSK1 at Thr581 between CNE1G and CNE1GL cells by immunocytochemical staining. CNE1GL cells stably expressed LMP1 showed substantially high level of MSK1 phosphorylation compared with control 


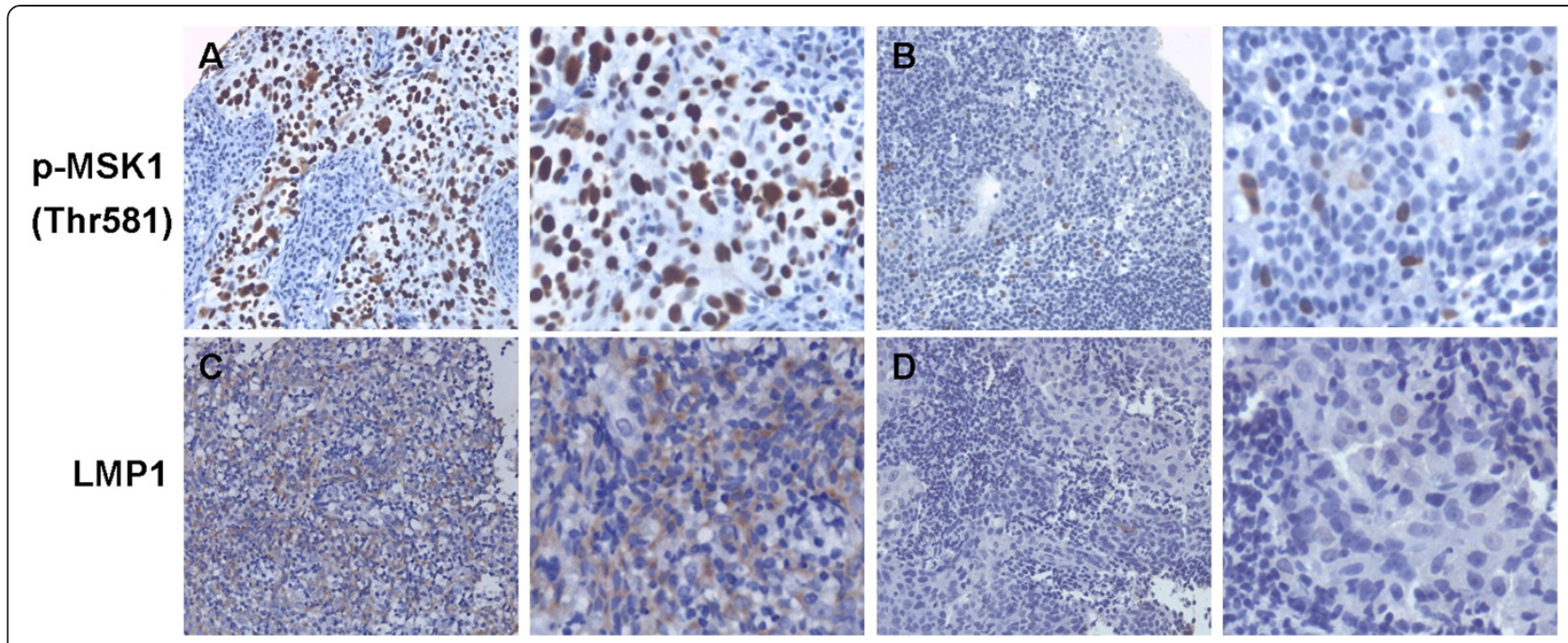

Fig. 1 Expressions of phosphorylated MSK1 (Thr581) and LMP1 in nasopharyngeal carcinoma (NPC) and normal nasopharynx tissues by immunohistochemistry. (a) High expression of phosphorylated MSK1 (Thr581) in poorly differentiated NPC tissues. (b) Low expression of phosphorylated MSK1 (Thr581) in normal nasopharynx tissues. (c) Positive expression of LMP1 in poorly differentiated NPC tissues. (d) Negative expression of LMP1 in poorly differentiated NPC tissues. Images were captured at $\times 200$ magnification (left) and $\times 400$ magnification (right)

CNE1G cells in the serum-starved condition (Fig. 2a), which was consistent with previous findings on MSK1 kinase activity [18]. In addition, CNE1 cells were transiently transfected with pcDNA3.0-LMP1, and the expression of phosphorylated MSK1 at Thr581 was examined by western blotting analysis. As shown in Fig. 2b, EBV-LMP1 could induce a dramatic increase of MSK1 phosphorylation at Thr581 in CNE1 cells. To further explore the signal mechanism mediated the activation of MSK1, PD98059, a specific ERK1/2 inhibitor, was used to treat the LMP1transfected CNE1 cells. Our results showed that PD98059 obviously suppressed LMP1-induced phosphorylation of MSK1 at Thr581 in a dose-dependent manner (Fig. 2c).

\section{H89 inhibits LMP1-promoted CNE1 cell proliferation by delaying $G_{1} / S$ transition}

To determine whether MSK1 activity is crucial for LMP1promoted cell proliferation in NPC cells, H89, a potent inhibitor of MSK1, was used to treat the LMP1-transfected CNE1 cells. As expected, H89 markedly inhibited the LMP1-induced phosphorylation of MSK1 at Thr581 in a dose-dependent manner, but the total MSK1 protein level did not change (Fig. 3a). We assessed the effect of H89 on

Table 1 The level of phosphorylated MSK1 (Thr581) in nasopharyngeal carcinoma (NPC) and normal nasopharynx tissues

\begin{tabular}{lccccr}
\hline Sample & $\mathrm{n}$ & \multicolumn{2}{c}{$\mathrm{p}$-MSK1 } & P-value \\
\cline { 3 - 4 } & & High & Low & \\
\hline poorly differentiated NPC & 53 & 29 & 24 & $<0.001$ \\
normal nasopharynx & 36 & 5 & 21 & \\
\hline
\end{tabular}

LMP1-promoted cell proliferation with different concentrations of H89 over varying periods. The results showed that H89 effectively suppressed LMP1-promoted cell proliferation in a time- and dose-dependent manner (Fig. 3b). In addition, H89 was found to dramatically block the colony formation promoted by LMP1 (Fig. 3c). The effect of H89 on cell cycle distributions was analyzed by flow cytometry. As shown in Fig. 3d, CNE1 cells expressing LMP1 showed a decrease of cells in $G_{0} / G_{1}$ phase, and an increase of cells in the $S$ and $G_{2} / M$ phases compared with mock cells, which indicated that LMP1 can accelerate cell cycle progression to promote the proliferation of $\mathrm{CNE} 1$ cells. However, the treatment of H89 caused a great accumulation of cells in the $G_{0} / G_{1}$ phase and a decrease in the $\mathrm{S}$ phase in a dose-dependent manner. These results suggested that H89 suppressed LMP1-promoted cell proliferation because of an impaired $G_{1} / S$ cell cycle transition.

\section{Knockdown of MSK1 suppresses LMP1-promoted CNE1 cell proliferation and anchorage-independent growth}

To ensure that the inhibition of cell proliferation was not a consequence of some other effect of H89, we designed

Table 2 Correlation between LMP1 expression and phosphorylated MSK1 (Thr581) in nasopharyngeal carcinoma (NPC) tissues

\begin{tabular}{|c|c|c|c|c|c|}
\hline \multirow[t]{2}{*}{ Groups } & & \multicolumn{2}{|c|}{ LMP1 } & \multirow[t]{2}{*}{$r$} & \multirow[t]{2}{*}{$P$-value } \\
\hline & & + & - & & \\
\hline \multirow[t]{2}{*}{ p-MSK1 } & high & 22 & 7 & 0.393 & 0.002 \\
\hline & low & 8 & 16 & & \\
\hline
\end{tabular}




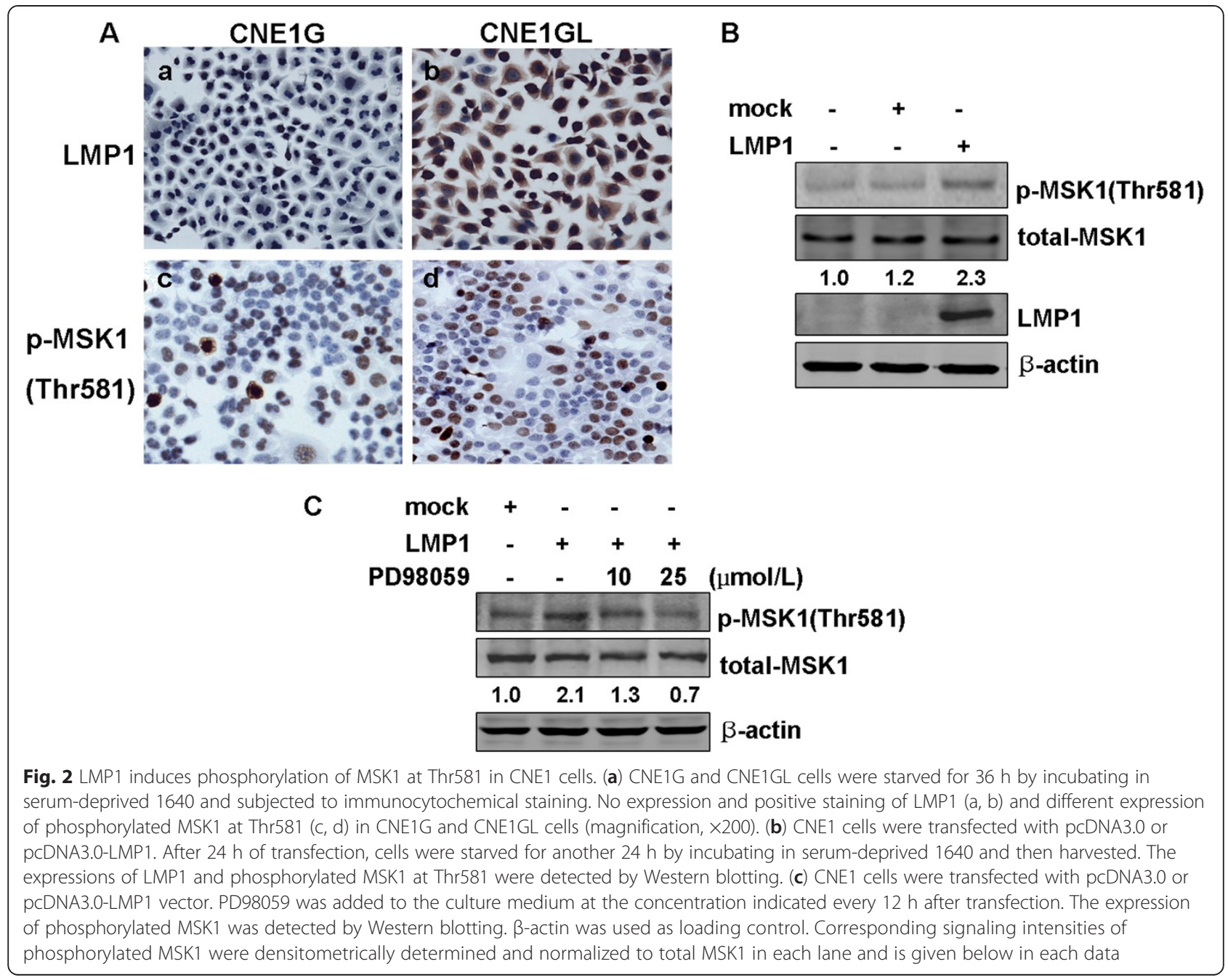

siRNA against MSK1 (si-MSK1) and a scrambled control siRNA (si-mock) for transfection into CNE1 cells. Immunoblot analysis revealed that CNE1 cells stably transfected with si-MSK1 showed a suppression of endogenous MSK1 expression of up to $80 \%$ compared with si-mock control cells (Fig. 4a). The si-mock or si-MSK1 stable CNE1 cells were transfected with pcDNA3.0 or pcDNA3.0-LMP1, and then cell proliferation and cell cycle profile were analyzed. Consistent to the effect of treatment with H89, the knockdown of MSK1 (si-MSK1/LMP1) markedly suppressed LMP1-promoted cell proliferation, and caused cell cycle arrest at the $G_{0} / G_{1}$ phase compared with the simock control cells (si-mock/LMP1) (Fig. 4b and 4c). Furthermore, the effect of MSK1 knockdown on anchorageindependent cell transformation was evaluated by soft agar assay. As shown in Fig. 4d, the knockdown of MSK1 strongly blocked the formation of transformed colonies induced by LMP1 compared with si-mock control cells. The inhibition was obvious not only in colony number, but also in colony size. These data indicated that MSK1 activity was required for LMP1-promoted malignant phenotype of CNE1 cells.

\section{MSK1 regulates LMP1-induced expressions of Fra-1 and c- Jun in CNE1 cells}

MSK1 has been showed to play an important role in transcriptional and epigenetic regulation of genes in response to extracellular stimuli. We further explored whether the heightened activity of MSK1 is responsible for LMP1-induced gene activation involved in cell proliferation and transformation. LMP1 was found to upregulate activator protein (AP-1) transcriptional activity [24], but its mechanisms involved are still not completely clear. Examining the expressions of AP-1 proteins, we found that LMP1 can increase the expressions of endogenous Fra-1 and c-Jun proteins. However, the treatment of H89 dramatically suppressed the promoter activity of Fra-1 or c-Jun induced by LMP1 in a dose- 


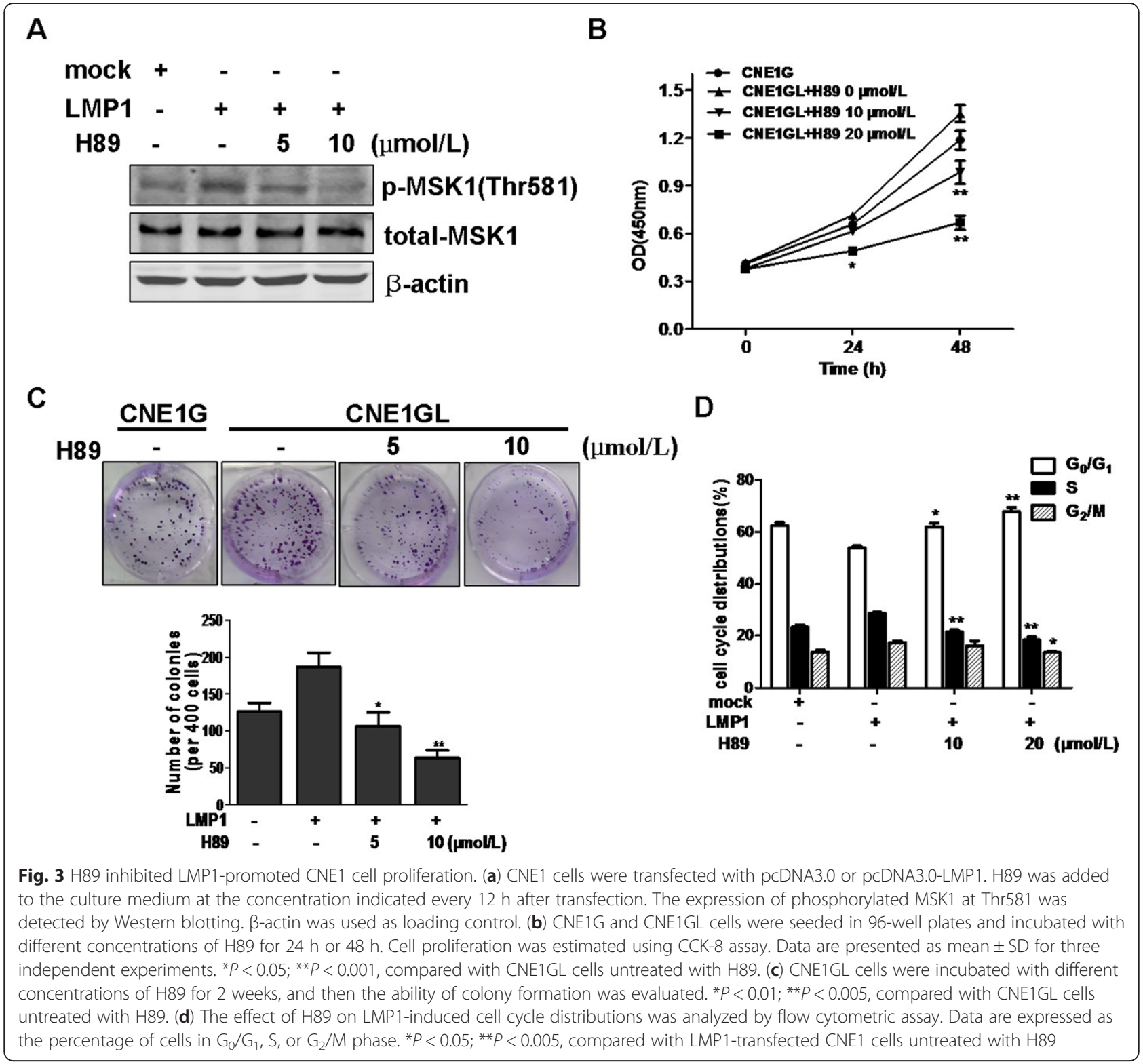

dependent manner (Fig. 5a), resulting in their decreasing protein levels (Fig. 5b). Next, we examined the effect of MSK1 knockdown on the induction of Fra-1 or c-Jun gene. As shown in Fig. 5c and 5d, LMP1-promoted induction of Fra-1 and c-Jun was effectively abrogated in si-MSK1 stably transfected cells compared with si-mock control cells. Furthermore, AP-1 activation induced by LMP1 was significantly blocked when MSK1 activity or expression was reduced in CNE1 cells, consistent to that observed for Fra-1 or c-Jun activity. Taken together, these results showed that the increased MSK1 activity in LMP1-transformed CNE1 cells is critically important for induction of Fra-1, c-Jun promoter and AP-1 activation.
Phosphorylation of histone $\mathrm{H} 3$ at Ser10 is involved in LMP1 induction of Fra-1 and c-Jun in CNE1 cells

It was proposed that increased phosphorylation of histone $\mathrm{H} 3$ at Ser10 by activation of Ras-MAPK pathway and MSK1 may contribute to the aberrant gene expression observed in oncogene-transformed cells [25]. Our previous studies showed that MSK1 mediated LMP1-induced phosphorylation of histone $\mathrm{H} 3$ at Ser10 in CNE1 cells [18]. Thus, we further determined whether phosphorylation of histone H3 at Ser10 is involved in LMP1-promoted activation of Fra-1 or c-Jun. The pcDNA6.0-H3 WT or mutant pcDNA6.0-H3 S10A plasmid was introduced into CNE1 cells (Fig. 6a), then the LMP1-promoted Fra-1 or c-Jun 


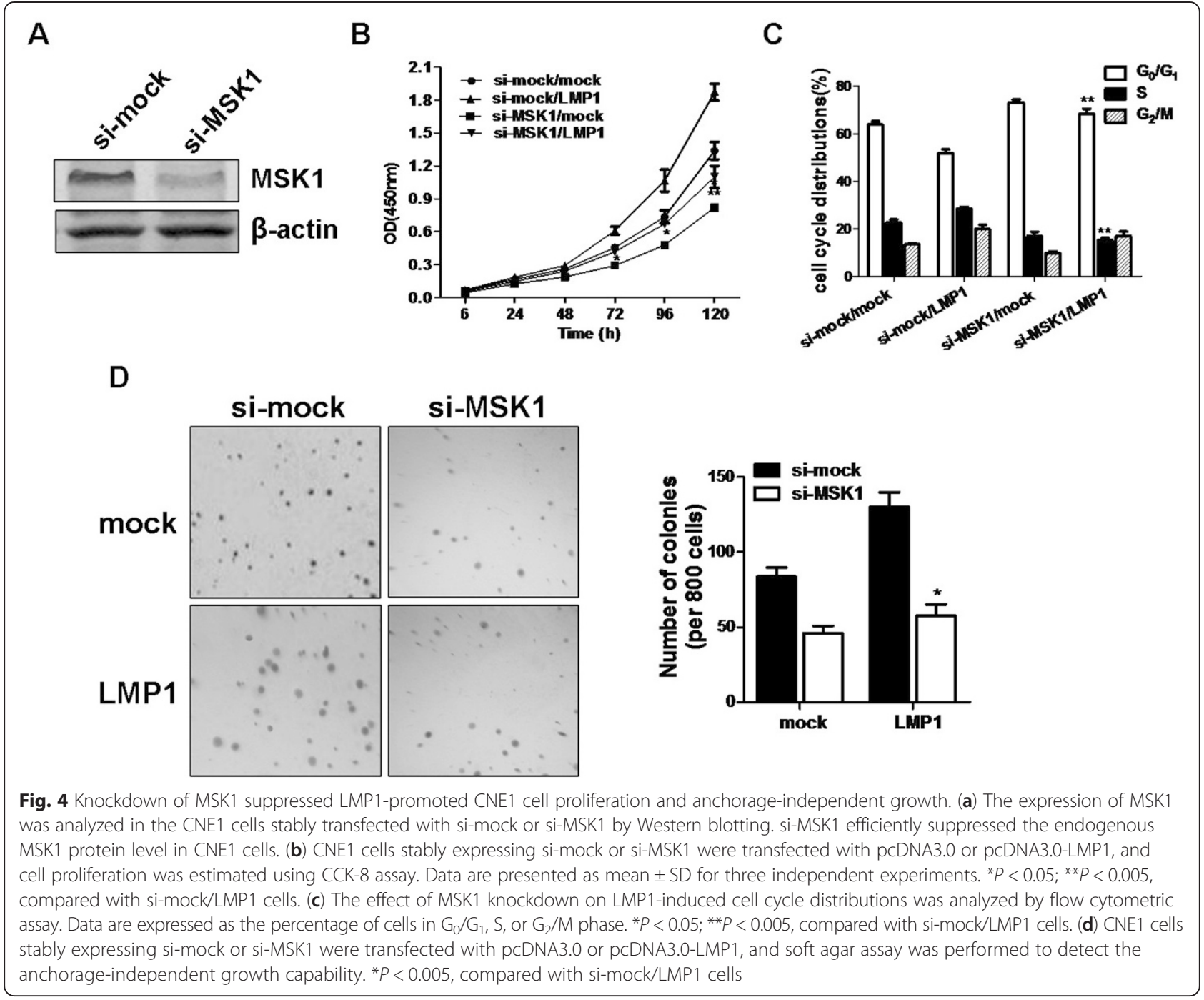

promoter activity was assessed. As shown in Fig. 6b, LMP1-promoted transcriptional activity of Fra-1 or c-Jun was significantly increased in CNE1 cells overexpressing H3 WT compared with mock control cells. In contrast, the histone H3 S10A mutant partly suppressed LMP1promoted induction of Fra-1 and c-Jun genes compared with H3 WT cells. We next detected the effect of histone $\mathrm{H} 3$ on the expression of Fra-1 or c-Jun proteins. Results showed that only H3 WT, but not H3 S10A, cells dramatically increased endogenous Fra-1 and c-Jun protein levels promoted by LMP1 compared with mock control cells (Fig. 6c). Taken together, these data suggested that histone H3 phosphorylation at Ser10 by MSK1 seems to be involved in LMP1-induced Fra-1 and c-Jun gene activation in CNE1 cells.

\section{Discussion}

MSK1 regulates transcription and cellular function at multiple levels and thereby exerts extensive effects in cell transformation, inflammation, neuronal plasticity, cardiac hypertrophy and other biological events [6]. MSK1 has been showed to be activated by either the ERK1/2 or p38 pathways depending on the specific stimuli. MSK1 activation is regulated by multiple phosphorylation sites. The phosphorylation at Thr581 located within the C-terminal kinase domain is essential for activation of MSK1. The mutation of Thr581 to an alanine residue prevented the activation of MSK1 in response to extracellular stimuli [26]. The elevated phosphoylation of MSK1 at Thr581 was observed in vulnerable neurons in Alzheimer's disease (AD) and the epidermis in lesional psoriatic skin, represented the activated ERK/p38 MAPK pathway and increased MSK1 activity [27, 28]. Various tumor promoters, such as EGF, TPA, or UVC and oncogenes have been showed to induce activation of MSK1 [29-31]. Furthermore, $\sim 30 \%$ of human cancers (e.g. colon, pancreatic, lung and breast cancers) have an overactive Ras-MAPK pathway and presumably elevated MSK1 activity even in 


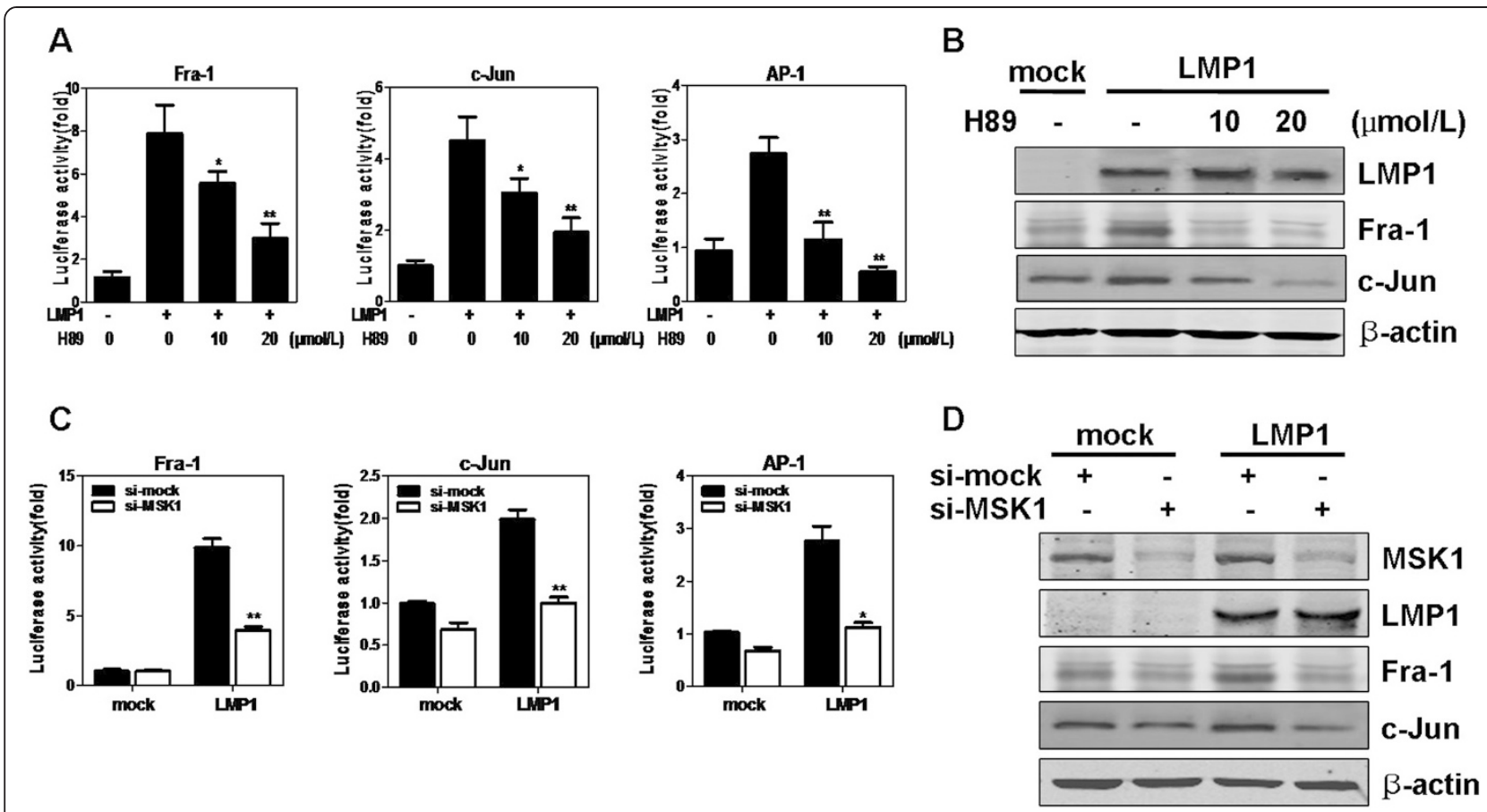

Fig. 5 MSK1 regulates LMP1-induced transcriptional activation of Fra-1 and c-Jun in CNE1 cells. (a) CNE1 cells were transfected with pcDNA3.0 or pcDNA3.0-LMP1. H89 was added to the culture medium at the concentration indicated every $12 \mathrm{~h}$ after transfection. The firefly luciferase activity was measured and normalized against Renilla luciferase activity respectively. Each bar represents the mean \pm SD of three independent experiments. ${ }^{*} P<0.05$; ${ }^{* *} P<0.005$, compared with LMP1-transfected CNE1 cells untreated with H89. (b) The effect of H89 on Fra-1 or c-Jun protein levels induced by LMP1 was examined by Western blotting. (c) CNE1 cells stably expressing si-mock or si-MSK1 were transfected with pCDNA3.0 or pcDNA3.0-LMP1. At 36 hours after transfection, the firefly luciferase activity was measured and normalized against Renilla luciferase activity respectively. ${ }^{*} P<0.01$; ${ }^{* *} P<0.005$, compared with si-mock/LMP1 cells. (d) The effect of MSK1 knockdown on Fra-1 or c-Jun protein levels induced by LMP1 was examined by Western blotting. $\beta$-actin was used as loading control

the absence of external stimuli $[14,15]$. In the present study, we found that the level of phosphorylated MSK1 at Thr581 was significantly high in poorly differentiated NPC tissues. It is indicated that the increased MSK1 activity might be an important event in the pathogenesis and progression of NPC.

LMP1, an important oncoprotein encoded by EBV, was found to be able to transform cell lines and induced multiple morphological and phenotypic alterations [4]. When expressed in epithelial cells, LMP1 exerted growth-promoting effects and modulated squamous epithelial differentiation [32, 33]. Functionally, LMP1 mimics a constitutively activated tumor necrosis factor receptor (TNFR), CD40, engaging a variety of signaling in a ligandindependent manner [34]. Activation of NF-kB, AP-1 and JAK3/STAT mediates various downstream pathological effects of LMP1 expression [5]. It has been demonstrated that LMP1 activates ERK-MAPK in epithelial cells via the canonical Raf-MEK-ERK-MAPK pathway in a Rasindependent manner [35]. In this study, we found that the elevated level of MSK1 phosphorylation at Thr581 in NPC tissues was closely related to LMP1 expression. Moreover, LMP1 induced phosphorylation of MSK1 at
Thr581 under the serum-starved condition in CNE1 cells. But ERK1/2 inhibitor PD98059 obviously suppressed LMP1-induced phosphorylation of MSK1. The findings suggested that EBV-LMP1 could constitutively activate MSK1 via ERK1/2-MAPK signaling pathway in NPC.

It has been reported that MSK1 played a positive role in the control of cell proliferation of $\mathrm{HaCaT}$ keratinocytes [36] and EGF- or TPA-induced neoplastic cell transformation of JB6 Cl41 cells [29]. The inhibition of MSK1 activation significantly suppressed malignant phenotype of Hras-transformed cells [13]. Furthermore, MSK1/2 knockout mice developed significantly fewer skin tumors compared with wild-type mice in DMBA-initiated and TPA-promoted multistage skin carcinogenesis [37]. In the present study, we found that MSK1 directly regulated LMP1-promoted cell proliferation and transformation in CNE1 cells. When expressed in CNE1 cells, LMP1 can exert growth-promoting effects through accelerating cell cycle progression. However, the inhibition of MSK1 activity or expression dramatically suppressed LMP1-promoted cell proliferation by causing cell cycle arrest at $\mathrm{G}_{1}-\mathrm{S}$ phase transition. The malignant potential of LMP1-transformed CNE1 cells, represented by the 


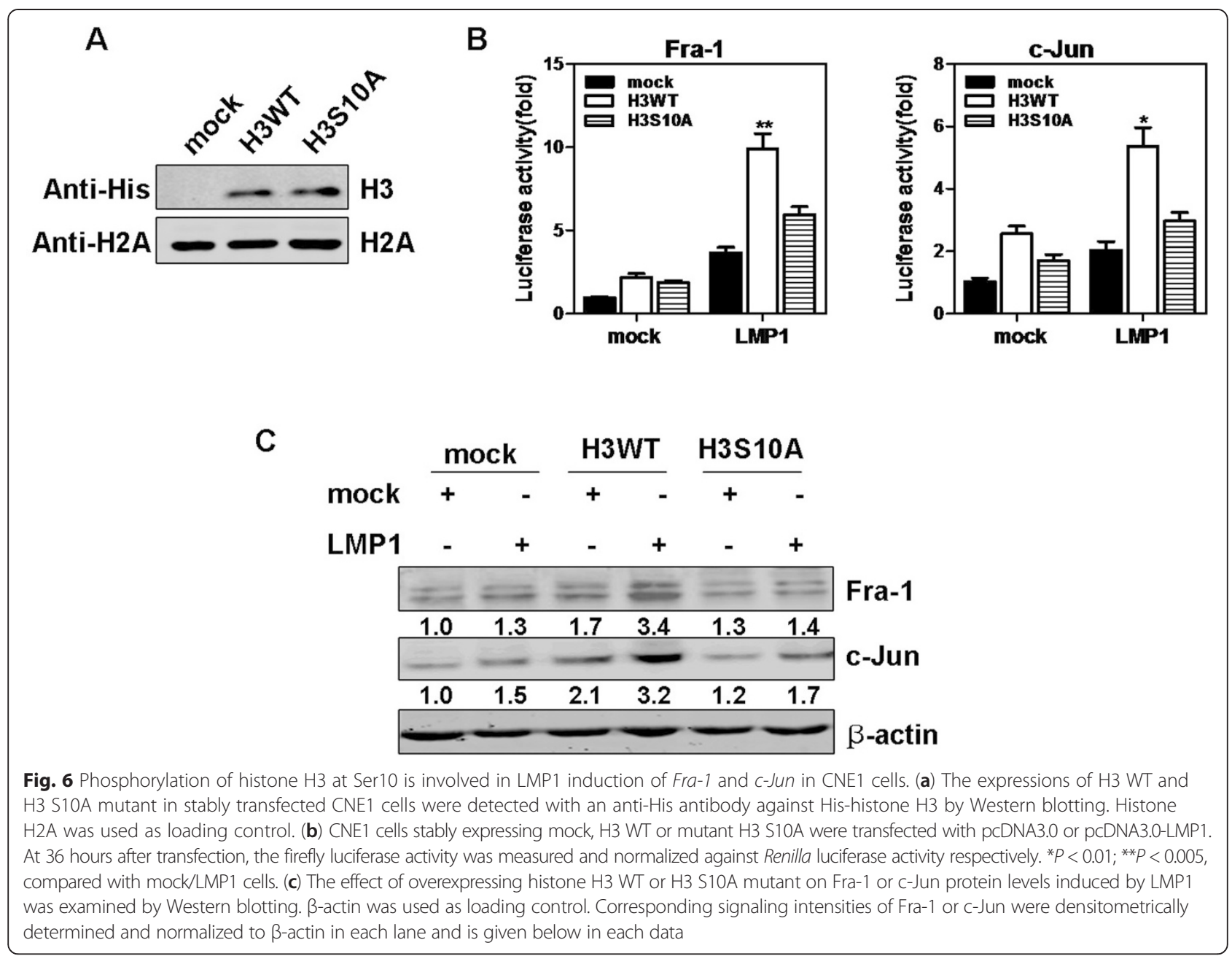

anchorage-independent cell growth capability, was reduced by the knockdown of MSK1. These results indicated that MSK1 was involved in cell cycle regulation and its activity was required for LMP1-promoted cell proliferation and transformation in CNE1 cells.

The role of MSK1 in cancer progression is varied because of their many substrates and encompasses involvement in cell proliferation, transformation and inflammation $[6,38]$. Increased MSK1 activity in ras-transformed mouse fibroblasts was required for maintaining the steady-state levels of Cox-2, Fra-1 and Jun and sustaining their ability to drive the cancer process [13]. MSK1 was involved in regulating the transcription of nuclear orphan receptor gene Nur77, Nurr1 and Nor1 [39], the up-regulation of which has been implicated in cell transformation [40]. The knockout of both MSK1 and MSK2 resulted in a $50 \%$ reduction in $c$ Fos and JunB gene transcription in response to anisomycin or UVC radiation, but the transcription of Egr-1 induced by anisomycin was not affected [7]. It has been reported that MSK1 activity was necessary for EGF- but not for TNF $\alpha$ - induced H3 Ser10 phosphorylation of the c-Fos promoter [41]. These studies strongly suggested that MSK1 might contribute to tumor promoter-induced cell transformation and tumorigenic progression through regulating the aberrant expression of specific genes. However, it remains to be resolved how MSK1 integrates the large variety of signals and accounts for the observed stimulus-, target gene- and cell-type-dependent specificity.

AP-1 is dimeric transcription factor mainly comprising members of Jun family (c-Jun、 JunB, and JunD) and Fos family (c-Fos、FosB、Fra-1, and Fra-2). AP-1 dimers of different composition exert significant difference in DNA binding affinity and determine the target genes that it regulates [42]. AP-1 proteins are primarily considered to be oncogenic and have been reported to involve in cell transformation, proliferation, differentiation and apoptosis $[42,43]$. The regulation of AP-1 proteins occurs at the level of transcription and/or post-transcriptional modifications. Fra-1, Fos-related antigen 1 encoding by Fosl1, dimerizes with Jun family proteins and thereby regulates 
various biological processes [44]. Fra-1 was strongly expressed in many kinds of epithelial tumors, and associated with poor prognosis of some squamous cell carcinomas [45, 46]. Fra-1 expression was also up-regulated in NPC cells compared with non-malignant nasopharyngeal epithelial cells [47]. Fra-1 was induced by EBV-LMP2A dependent on the activated ERK1/2 pathway and was essential for formation of AP-1 heterodimers mediating LMP2A-triggered MMP9 expression [48]. A potential link between LMP1 and Fra-1 comes from a previous study showing constitutive induction of Fra-1 in LMP1-transgenic mice [49]. c-Jun is a positive regulator of cell proliferation. The deficiency of c-Jun in fibroblasts leads to a marked proliferation defect due to the delay of cell cycle progression [50]. c-Jun played a pivotal role in Ras-induced cellular transformation and malignancy [51]. TNF- $\alpha$-stimulated c-Jun expression preceded and was essential for subsequent Fra-1 induction in pulmonary epithelial cells [52]. Previous studies have been shown that LMP1 promoted the formation of c-Jun/JunB heterodimers leading to induction of AP-1 activation through JNK-mediated phosphorylation of c-Jun [53]. In this study, we found that LMP1 caused a significant increase in the transcriptional activity of Fra-1 and c-Jun dependent on MSK1 activity. Chemical and genetic interference with MSK1 suppressed induction of Fra-1 and c-Jun by LMP1, resulting in decreased AP-1 activity. These findings indicated that MSK1 activity was critical in maintaining LMP1-promoted expressions of Fra-1 and c-Jun, which might alter AP-1 activation and its responsive genes.

Phosphorylation of histone $\mathrm{H} 3$ at Ser10 occurs in two difference phases of cell cycle with opposite functions. During mitosis, histone H3 Ser10 is globally phosphorylated by Aurora kinase, which facilitates compaction and transcriptional repression [54]. During interphase, extracellular stimuli also lead to phosphorylation of histone $\mathrm{H} 3$ in a minute fraction of histone $\mathrm{H} 3$, in correlation with rapid transcription induction of a subset of genes [55]. Accumulating evidences have demonstrated that phosphorylation of histone H3 at Ser10 in interphase is involved in different signaling pathways and effector kinases depending on specific stimulation and stress. Akt1, ERK2 and Ribosomal subunit protein S6 kinase 2 (RSK2), but not MSK1, were responsible for arseniteinduced phosphorylation of H3 at Ser10 [56], which was associated with induction of Fos, Egr1 and $I L-8$ [57]. It has been showed that PIM1 phosphorylated histone H3 at Ser10 on the nucleosome at the Myc-binding sites, contributing to transcriptional activation of Fra-1 gene [58]. Our previous study has revealed that LMP1 can constitutively activate phosphorylation of histone $\mathrm{H} 3$ at Ser10 in interphase through the activation of Ras-MAPK pathway and MSK1 kinase in CNE1 cells [18]. In this study, we found that overexpression of histone H3 WT in CNE1 cells dramatically augmented LMP1 induction of Fra-1 and c-Jun promoter activity compared with mock control cells, but the increase of activity was partly inhibited in the mutant H3 S10A cells. Constitutive activation of ERK-MSK1 pathway induced by LMP1 enhanced the level of phosphorylated histone $\mathrm{H} 3$ at Ser10 in H3 WT-overexpressing CNE1 cells. The elevated histone $\mathrm{H} 3$ phosphorylation may contribute to the decondensed chromatin struction and releasing blocks in elongation, which promotes the binding of transcription factors to the promoter region leading to the aberrant transcriptional activation of genes $[55,59]$. The regulation of Fra-1 or c-Jun gene is orchestrated by numerous transcription factors, which themselves must be activated. Moreover, other post-translational modifications of histone $\mathrm{H} 3$ such as methylation and acetylation might also be involved. Nonetheless, it has been suggested that phosphorylation of histone $\mathrm{H} 3$ at Ser10 mediated by MSK1 was closely linked with LMP1-induced transcriptional activation of Fra-1 and c-Jun genes.

\section{Conclusions}

In summary, these studies demonstrated a pivotal role of MSK1-mediated nucleosomal response in LMP1-promoted cell proliferation and transformation in NPC, which might be associated with its induction of aberrant gene expression. Considering that MSK1 is the active link between signaling cascades and the primary response of gene expression. It has been showed that MSK1/2 knock-out mice are viable and fertile and have no obvious health defects [7]. Therefore, MSK1 may consider as an ideal molecular target for cancer chemotherapy and gene therapy in NPC.

\section{Abbreviations \\ NPC: Nasopharyngeal carcinoma; EBV: Epstein-Barr virus; LMP1: Latent memberane protein 1; MSK1: Mitogen- and stress-activated kinase 1; AP-1: Activator protein-1; Fra-1: Fos-related antigen 1; siRNA: Small interfering RNA.}

\section{Competing interests}

The authors declare that they have no competing interests.

\section{Authors' contributions}

BB $L$ designed and performed the experiments, analyzed data and drafted the manuscript. G H, Z H and X Z contributed reagents, performed experiments and analyzed data. $Z$ W, D L and $S L$ performed experiments and analyzed data. $\mathrm{Z} \mathrm{H}$ designed the experiments, analyzed data and drafted the manuscript. All authors read and approved the final manuscript.

\section{Acknowledgments}

This work was supported by PhD Start-up Fund of Guangdong medical college (No: B2014001 to BB L) and National Natural Science Foundation of China (No: 81372137 to ZW H). The authors are grateful for the support from Dr. Xiaoyi Chen, Cancer Research Institute, Guangdong Medical College, Guangdong, China; Dr. Zigang Dong, Hormel Institute, University of Minnesota, Austin, Minnesota, USA.

Received: 10 December 2014 Accepted: 29 April 2015 Published online: 10 May 2015

\section{References}

1. Chang ET, Adami HO. The enigmatic epidemiology of nasopharyngeal carcinoma. Cancer Epidemiol Biomarkers Prev. 2006;15(10):1765-77. 
2. Tsao SW, Yip YL, Tsang CM, Pang PS, Lau VM, Zhang G, et al. Etiological factors of nasopharyngeal carcinoma. Oral Oncol. 2014;50(5):330-8.

3. Raab-Traub N. Epstein-Barr virus in the pathogenesis of NPC. Semin Cancer Biol. 2002;12(6):431-41.

4. Hu LF, Chen F, Zheng X, Ernberg I, Cao SL, Christensson B, et al. Clonability and tumorigenicity of human epithelial cells expressing the EBV encoded membrane protein LMP1. Oncogene. 1993;8(6):1575-83.

5. Eliopoulos AG, Young LS. LMP1 structure and signal transduction. Semin Cancer Biol. 2001;11(6):435-44.

6. Vermeulen L, Vanden Berghe W, Beck IM, De Bosscher K, Haegeman G. The versatile role of MSKs in transcriptional regulation. Trends Biochem Sci. 2009;34(6):311-8.

7. Wiggin GR, Soloaga A, Foster JM, Murray-Tait V, Cohen P, Arthur JS. MSK1 and MSK2 are required for the mitogen- and stress-induced phosphorylation of CREB and ATF1 in fibroblasts. Mol Cell Biol. 2002;22(8):2871-81.

8. Vermeulen L, De Wilde G, Van Damme P, Vanden Berghe W Haegeman G. Transcriptional activation of the NF-kappaB p65 subunit by mitogen- and stress-activated protein kinase-1 (MSK1). EMBO J. 2003;22(6):1313-24.

9. Zhang Y, Liu G, Dong Z. MSK1 and JNKs mediate phosphorylation of STAT3 in UVA-irradiated mouse epidermal JB6 cells. J Biol Chem. 2001;276(45):42534-42

10. Soloaga A, Thomson S, Wiggin GR, Rampersaud N, Dyson MH, Hazzalin CA, et al. MSK2 and MSK1 mediate the mitogen- and stress-induced phosphorylation of histone $\mathrm{H3}$ and HMG-14. EMBO J. 2003;22(11):2788-97.

11. Chadee DN, Hendzel MJ, Tylipski CP, Allis CD, Bazett-Jones DP, Wright JA, et al. Increased Ser-10 phosphorylation of histone $\mathrm{H} 3$ in mitogen-stimulated and oncogene-transformed mouse fibroblasts. J Biol Chem. 1999;274(35):24914-20.

12. Choi HS, Choi BY, Cho YY, Mizuno H, Kang BS, Bode AM, et al Phosphorylation of histone $\mathrm{H} 3$ at serine 10 is indispensable for neoplastic cell transformation. Cancer Res. 2005;65(13):5818-27.

13. Perez-Cadahia B, Drobic B, Espino PS, He S, Mandal S, Healy S, et al. Role of MSK1 in the malignant phenotype of Ras-transformed mouse fibroblasts. J Biol Chem. 2011;286(1):42-9.

14. Dunn KL, Espino PS, Drobic B, He S, Davie JR. The Ras-MAPK signal transduction pathway, cancer and chromatin remodeling. Biochem Cell Biol. 2005;83(1):1-14

15. Calipel A, Lefevre G, Pouponnot C, Mouriaux F, Eychene A, Mascarelli F. Mutation of B-Raf in human choroidal melanoma cells mediates cell proliferation and transformation through the MEK/ERK pathway. J Biol Chem. 2003;278(43):42409-18.

16. Espino PS, Li L, He S, Yu J, Davie JR. Chromatin modification of the trefoil factor 1 gene in human breast cancer cells by the Ras/mitogen-activated protein kinase pathway. Cancer Res. 2006;66(9):4610-6.

17. Sundar IK, Chung S, Hwang JW, Lapek Jr JD, Bulger M, Friedman AE, et al. Mitogen- and stress-activated kinase 1 (MSK1) regulates cigarette smoke-induced histone modifications on NF-kappaB-dependent genes. PLoS One. 2012;7(2):e31378

18. Li B, Huang G, Zhang X, Li R, Wang J, Dong Z, et al. Increased phosphorylation of histone $\mathrm{H} 3$ at serine 10 is involved in Epstein-Barr virus latent membrane protein-1-induced carcinogenesis of nasopharyngeal carcinoma. BMC Cancer. 2013;13:124.

19. Chen $Y$, Chen XY. Effect of Epstein-Barr virus latent membrane protein 1 (LMP1) on apoptosis of nasopharyngeal carcinoma cell line CNE1. Ai Zheng. 2002:21(5):498-503.

20. Kieser A, Kilger E, Gires O, Ueffing M, Kolch W, Hammerschmidt W. Epstein-Barr virus latent membrane protein-1 triggers AP-1 activity via the c-Jun N-terminal kinase cascade. EMBO J. 1997;16(21):6478-85.

21. Adiseshaiah P, Papaiahgari SR, Vuong H, Kalvakolanu DV, Reddy SP. Multiple cis-elements mediate the transcriptional activation of human fra-1 by 12-O-tetradecanoylphorbol-13-acetate in bronchial epithelial cells. J Biol Chem. 2003:278(48):47423-33.

22. Shin HM, Han TH. CD28-mediated regulation of the c-jun promoter involves the MEF2 transcription factor in Jurkat T cells. Mol Immunol. 1999;36(3):197-203.

23. Horikawa T, Sheen TS, Takeshita H, Sato H, Furukawa M, Yoshizaki T Induction of c-Met proto-oncogene by Epstein-Barr virus latent membrane protein-1 and the correlation with cervical lymph node metastasis of nasopharyngeal carcinoma. Am J Pathol. 2001;159(1):27-33.
24. Deng $L$, Yang J, Zhao XR, Deng XY, Zeng $L$, Gu HH, et al. Cells in G2/M phase increased in human nasopharyngeal carcinoma cell line by EBV-LMP1 through activation of NF-kappaB and AP-1. Cell Res. 2003;13(3):187-94.

25. Strelkov IS, Davie JR. Ser-10 phosphorylation of histone $\mathrm{H} 3$ and immediate early gene expression in oncogene-transformed mouse fibroblasts. Cancer Res. 2002;62(1):75-8.

26. McCoy CE, Campbell DG, Deak M, Bloomberg GB, Arthur JS. MSK1 activity is controlled by multiple phosphorylation sites. Biochem J. 2005;387(Pt 2):507-17.

27. Webber KM, Smith MA, Lee HG, Harris PL, Moreira P, Perry G, et al. Mitogenand stress-activated protein kinase 1: convergence of the ERK and p38 pathways in Alzheimer's disease. J Neurosci Res. 2005;79(4):554-60.

28. Funding AT, Johansen C, Kragballe K, Otkjaer K, Jensen UB, Madsen MW, et al. Mitogen- and stress-activated protein kinase 1 is activated in lesional psoriatic epidermis and regulates the expression of pro-inflammatory cytokines. J Invest Dermatol. 2006;126(8):1784-91.

29. Kim HG, Lee KW, Cho YY, Kang NJ, Oh SM, Bode AM, et al. Mitogen- and stress-activated kinase 1-mediated histone $\mathrm{H} 3$ phosphorylation is crucial for cell transformation. Cancer Res. 2008;68(7):2538-47.

30. Tange S, Ito S, Senga T, Hamaguchi M. Phosphorylation of histone H3 at Ser 10: its role in cell transformation by v-Src. Biochem Biophys Res Commun. 2009:386(4):588-92.

31. Drobic B, Espino PS, Davie JR. Mitogen- and stress-activated protein kinase 1 activity and histone h3 phosphorylation in oncogene-transformed mouse fibroblasts. Cancer Res. 2004;64(24):9076-9.

32. Fahraeus R, Rymo L, Rhim JS, Klein G. Morphological transformation of human keratinocytes expressing the LMP gene of Epstein-Barr virus. Nature. 1990;345(6274):447-9.

33. Dawson CW, Rickinson AB, Young LS. Epstein-Barr virus latent membrane protein inhibits human epithelial cell differentiation. Nature. 1990;344(6268):777-80

34. Mosialos G, Birkenbach M, Yalamanchili R, VanArsdale T, Ware C, Kieff E. The Epstein-Barr virus transforming protein LMP1 engages signaling proteins for the tumor necrosis factor receptor family. Cell. 1995;80(3):389-99.

35. Dawson CW, Laverick L, Morris MA, Tramoutanis G, Young LS. Epstein-Barr virus-encoded LMP1 regulates epithelial cell motility and invasion via the ERK-MAPK pathway. J Virol. 2008:82(7):3654-64.

36. Schiller M, Bohm M, Dennler S, Ehrchen JM, Mauviel A. Mitogen- and stressactivated protein kinase 1 is critical for interleukin-1-induced, CREB-mediated, c-fos gene expression in keratinocytes. Oncogene. 2006;25(32):4449-57.

37. Chang S, Iversen L, Kragballe K, Arthur JS, Johansen C. Mice lacking MSK1 and MSK2 show reduced skin tumor development in a two-stage chemical carcinogenesis model. Cancer Invest. 2011;29(3):240-5.

38. Davie JR, Drobic B, Perez-Cadahia B, He S, Espino PS, Sun JM, et al. Nucleosomal response, immediate-early gene expression and cell transformation. Adv Enzyme Regul. 2010;50(1):135-45.

39. Darragh J, Soloaga A, Beardmore VA, Wingate AD, Wiggin GR, Peggie M, et al. MSKs are required for the transcription of the nuclear orphan receptors Nur77, Nurr1 and Nor1 downstream of MAPK signalling. Biochem J. 2005;390(Pt 3):749-59.

40. Ke N, Claassen G, Yu DH, Albers A, Fan W, Tan P, et al. Nuclear hormone receptor NR4A2 is involved in cell transformation and apoptosis. Cancer Res. 2004;64(22):8208-12.

41. Duncan EA, Anest V, Cogswell P, Baldwin AS. The kinases MSK1 and MSK2 are required for epidermal growth factor-induced, but not tumor necrosis factor-induced, histone H3 Ser10 phosphorylation. J Biol Chem. 2006:281(18):12521-5.

42. Bakiri L, Matsuo K, Wisniewska M, Wagner EF, Yaniv M. Promoter specificity and biological activity of tethered AP-1 dimers. Mol Cell Biol. 2002;22(13):4952-64.

43. Angel $\mathrm{P}$, Karin M. The role of Jun, Fos and the AP-1 complex in cell-proliferation and transformation. Biochim Biophys Acta. 1991;1072(2-3):129-57.

44. Cohen DR, Ferreira PC, Gentz R, Franza Jr BR, Curran T. The product of a fos-related gene, fra-1, binds cooperatively to the AP-1 site with Jun: transcription factor AP-1 is comprised of multiple protein complexes. Genes Dev. 1989;3(2):173-84.

45. Zhang L, Pan HY, Zhong LP, Wei KJ, Yang X, Li J, et al. Fos-related activator1 is overexpressed in oral squamous cell carcinoma and associated with tumor lymph node metastasis. J Oral Pathol Med. 2010;39(6):470-6.

46. Usui A, Hoshino I, Akutsu Y, Sakata H, Nishimori T, Murakami K, et al. The molecular role of Fra-1 and its prognostic significance in human esophageal squamous cell carcinoma. Cancer. 2012;118(13):3387-96. 
47. Fung LF, Lo AK, Yuen PW, Liu Y, Wang XH, Tsao SW. Differential gene expression in nasopharyngeal carcinoma cells. Life Sci. 2000;67(8):923-36.

48. Lan YY, Hsiao JR, Chang KC, Chang JS, Chen CW, Lai HC, et al. Epstein-Barr virus latent membrane protein $2 \mathrm{~A}$ promotes invasion of nasopharyngeal carcinoma cells through ERK/Fra-1-mediated induction of matrix metalloproteinase 9. J Virol. 2012;86(12):6656-67.

49. Charalambous CT, Hannigan A, Tsimbouri P, McPhee GM, Wilson JB. Latent membrane protein 1-induced EGFR signalling is negatively regulated by TGF alpha prior to neoplasia. Carcinogenesis. 2007;28(8):1839-48.

50. Schreiber M, Kolbus A, Piu F, Szabowski A, Mohle-Steinlein U, Tian J, et al. Control of cell cycle progression by c-Jun is p53 dependent. Genes Dev. 1999;13(5):607-19.

51. Johnson R, Spiegelman B, Hanahan D, Wisdom R. Cellular transformation and malignancy induced by ras require c-jun. Mol Cell Biol. 1996;16(8):4504-11.

52. Adiseshaiah P, Kalvakolanu DV, Reddy SP. A JNK-independent signaling pathway regulates TNF alpha-stimulated, c-Jun-driven FRA-1 protooncogene transcription in pulmonary epithelial cells. J Immunol. 2006;177(10):7193-202.

53. Song $X$, Tao $Y G$, Deng $X Y$, Jin $X$, Tan $Y N$, Tang $M$, et al. Heterodimer formation between c-Jun and Jun B proteins mediated by Epstein-Barr virus encoded latent membrane protein 1. Cell Signal. 2004;16(10):1153-62.

54. Crosio C, Fimia GM, Loury R, Kimura M, Okano Y, Zhou H, et al. Mitotic phosphorylation of histone $\mathrm{H} 3$ : spatio-temporal regulation by mammalian Aurora kinases. Mol Cell Biol. 2002;22(3):874-85.

55. Nowak SJ, Corces VG. Phosphorylation of histone H3: a balancing act between chromosome condensation and transcriptional activation. Trends Genet. 2004;20(4):214-20.

56. He Z, Ma WY, Liu G, Zhang Y, Bode AM, Dong Z. Arsenite-induced phosphorylation of histone $\mathrm{H} 3$ at serine 10 is mediated by Akt1, extracellular signal-regulated kinase 2, and p90 ribosomal 56 kinase 2 but not mitogenand stress-activated protein kinase 1. J Biol Chem. 2003;278(12):10588-93.

57. Suzuki T, Kita K, Ochi T. Phosphorylation of histone $\mathrm{H} 3$ at serine 10 has an essential role in arsenite-induced expression of FOS, EGR1 and IL8 mRNA in cultured human cell lines. J Appl Toxicol. 2013;33(8):746-55.

58. Zippo A, De Robertis A, Serafini R, Oliviero S. PIM1-dependent phosphorylation of histone $\mathrm{H} 3$ at serine 10 is required for MYC-dependent transcriptional activation and oncogenic transformation. Nat Cell Biol. 2007;9(8):932-44.

59. Sawicka A, Seiser C. Histone H3 phosphorylation - a versatile chromatin modification for different occasions. Biochimie. 2012;94(11):2193-201.

\section{Submit your next manuscript to BioMed Central and take full advantage of:}

- Convenient online submission

- Thorough peer review

- No space constraints or color figure charges

- Immediate publication on acceptance

- Inclusion in PubMed, CAS, Scopus and Google Scholar

- Research which is freely available for redistribution 